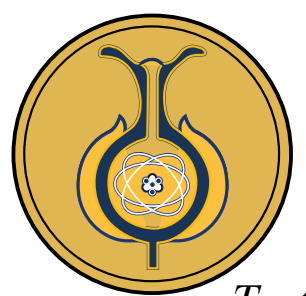

\title{
Selección clonal de Tectona grandis L. f. para el Pacífico seco de Costa Rica
}

Tectona grandis L.f. clonal selection for the dry Pacific zone of Costa Rica

Seleção clonal de Tectona grandis L. f. para o Pacífico Seco da Costa Rica

Yorleny Badilla-Valverde ${ }^{1,2}$, Olman Murillo-Gamboa ${ }^{1}$

Received: Dec/18/2020 • Accepted: Nov/11/2021 • Published: Feb/14/2022

\section{Resumen (10)}

La teca es una de las especies comerciales plantadas en el trópico con mayor valor en el mundo. La cooperativa internacional de mejoramiento genético GENFORES, establecida en Costa Rica, ha liderado el mejoramiento genético de la especie en América Latina. El estudio se realizó con el objetivo de seleccionar los clones con mayor adaptabilidad específica al Pacífico seco de Costa Rica y de otras regiones tropicales secas. La investigación se desarrolló en Nicoya, Guanacaste (Pacífico norte de Costa Rica), donde se evaluaron 31 genotipos durante 13 años continuos (2006-2019), con base en el crecimiento en diámetro, volumen comercial y la calidad del fuste. Mediante el software Selegen-REML/BLUP se obtuvieron los parámetros genéticos, el ranking de selección y la ganancia genética estimada. A los 8,9 años fue la edad más temprana donde se registraron los valores más altos de heredabilidad individual y de heredabilidad media clonal para el diámetro $\left(h^{2} g=0,12\right.$ y $\left.h^{2} m c=0,82\right)$ y para el volumen comercial $\left(h^{2} g=0,075 y h^{2} m c\right.$ $=0,74)$. Plantaciones comerciales establecidas con los mejores 10 clones, a los 12,9 años de edad podrían alcanzar un volumen comercial de $0,88 \mathrm{~m}^{3} a ́ r b o l^{-1}$, que corresponde a una ganancia genética de un $11 \%$ con respecto a la mejor semilla comercial disponible del huerto semillero. El diámetro alcanzaría $28 \mathrm{~cm} \mathrm{a}$ esta misma edad. Tales resultados permitirán reducir cerca de 2 años la edad de cosecha de plantaciones de teca en el Pacífico seco del país.

Palabras clave: ganancia genética; mejoramiento genético; Guanacaste; silvicultura clonal; teca.

\section{Abstract (1)}

Teak has the highest value of any commercially planted tree species in the tropics. The tree improvement international cooperative GENFORES, established in Costa Rica, has led breeding programs with this tree species in Latin America. This investigation was undertaken with the objective of selecting the clones which are best adapted to the dry Pacific zone of Costa Rica, as well as for other dry regions in the tropics. Research was carried out in Nicoya, in the province of Guanacaste (in the northern Pacific region of Costa Rica), where 31 genotypes were evaluated for 13 continuous years, in terms of diameter growth, commercial volume, and stem quality. Genetic parameters were obtained using Selegen-REML/BLUP software, as were selection

Yorleny Badilla-Valverde, $\$ yorlenybadilla@yahoo.es, (D) https://orcid.org/0000-0002-6743-9734

Olman Murillo-Gamboa, \olmuga@yahoo.es, (D https://orcid.org/0000-0003-3213-8867

1 Escuela de Ingeniería Forestal, Instituto Tecnológico de Costa Rica, Cartago, Costa Rica.

2 Doctorado en Ciencias para el Desarrollo (DOCINADE, Instituto Tecnológico de Costa Rica, Universidad Nacional de Costa Rica, Universidad Estatal a Distancia) San José, Costa Rica. 
rankings and estimated genetic gain. The highest values for individual heritability and mean clonal heritability for diameter $\left(h^{2} g=0.12\right.$ and $h^{2} m c=0.82$ ), and for commercial volume ( $h^{2} g=0.075$ and $h^{2} \mathrm{mc}$ $=0.74$ ) were obtained for trees that were 8.9 years old. Commercial plantations established with the best 10 clones, with an age of 12.9 years, may attain a commercial volume of $0.88 \mathrm{~m}^{3}$ per tree, corresponding to a genetic gain of $11 \%$ with respect to the best available seed source from a seed orchard, while diameter would reach $28 \mathrm{~cm}$ at the same age. These results allow a reduction of approximately two years of the age at which teak can be harvested in the dry Pacific zone of the country.

Key words: breeding; teak; clonal forestry; genetic gain; Guanacaste.

\section{Resumo (1)}

A teca é uma das espécies comerciais mais valiosas plantadas nos trópicos do mundo. A cooperativa internacional de melhoramento genético GENFORES, estabelecida na Costa Rica, liderou o melhoramento genético da espécie na América Latina. 0 estudo foi realizado com o objetivo de selecionar os clones com maior adaptabilidade específica no Pacífico Seco da Costa Rica e outras regiões tropicais secas. A pesquisa foi desenvolvida em Nicoya, Guanacaste (Pacífico Norte da Costa Rica), onde 31 genótipos foram avaliados ao longo de 13 anos contínuos (2006-2019), com base no crescimento do diâmetro, volume comercial e qualidade do eixo. Por meio do software Selegen-REML/BLUP, foram obtidos os parâmetros genéticos, 0 ranking de seleção e o ganho genético estimado. Aos 8,9 anos foi a idade mais antiga onde os maiores valores de hereditariedade individual e herdabilidade clonal média foram registrados para o diâmetro $\left(\mathrm{h}^{2} \mathrm{~g}\right.$ $=0,12$ e $\left.h^{2} m c=0,82\right)$ e para o volume comercial $\left(h^{2} g=0,075\right.$ e $\left.h^{2} m c=0,74\right)$. Plantações comerciais estabelecidas com os 10 melhores clones, com 12,9 anos de idade, poderiam atingir um volume comercial de $0,88 \mathrm{~m}^{3}$ árvore ${ }^{-1}, 0$ que corresponde a um ganho genético de $11 \%$ em relação à melhor semente comercial disponível do pomar de sementes. 0 diâmetro chegaria a $28 \mathrm{~cm}$ nesta mesma idade. Tais resultados permitirão a redução de cerca de dois anos a idade de colheita das plantações de teca no Pacífico Seco do país.

Palavras-chave: ganho genético; melhoramento genético; Guanacaste; silvicultura clonal; teca.

\section{Introducción}

La teca (Tectona grandis) ha sido una de las especies forestales de mayor interés en el mundo tropical y subtropical, lo cual motivó el inicio de trabajos de mejoramiento genético desde mediados del siglo anterior (Richens, 1945; Gram y Larsen, 1958; Keiding, 1960; Cameron, 1966). El alto valor de su madera en los mercados asiáticos y europeos ha promovido su plantación en numerosos países. Es muy probable que sea la especie más plantada en la región tropical del continente americano, donde fue introducida hace poco más de un siglo desde Myanmar a través de Trinidad y Tobago en 1913, en tiempos en que formaba parte del imperio británico (Keogh, 1979; 1980). Otras introducciones de semilla procedente de Sri Lanka (Ceylán) vía Panamá también han sido reportadas (Béhaghel, 1999). De las Antillas se distribuyó en pequeñas cantidades hacia Panamá, Costa Rica, Honduras, Ecuador y Colombia, por medio de las 
compañías bananeras. En Costa Rica, Fonseca (2004) relata que desde los años 20 la compañía bananera realizó varias introducciones pequeñas desde Trinidad y Tobago, pero ya en los años 50 se estableció una plantación comercial de más de 200 ha en la región de Quepos (Pacífico Central del país). Mientras que en Mato Grosso, Brasil, se reporta el ingreso de semilla de teca desde Trinidad y Tobago a finales de los años 60 (empresa Cáceres Florestal), de donde se constituyeron las primeras fuentes semilleras del país (Schuhli y Paludzyszyn, 2010). En los años 70 y 80, la importancia de la especie motivó al desarrollo de una intensa cooperación internacional, para el intercambio y establecimiento de ensayos de procedencias en una gran cantidad de países tropicales (Keiding, Wellendorf y Lauridsen, 1986; Dupuy y Verhaegen, 1993). En años recientes, varias compañías privadas en Costa Rica y en Brasil continuaron introduciendo procedencias nativas asiáticas, así como procedencias derivadas desde Malasia y Tanzania (Murillo, Wright, Monteuuis y Montenegro, 2013), algunas de ellas con buen suceso en términos de productividad (Goh y Monteuuis 2005; Goh, Chaix, Bailleres y Monteuuis, 2007).

La posibilidad de clonar árboles de teca se reporta desde finales del siglo anterior mediante la tecnología de cultivo in vitro (Mascarenhas, Kendurkar y Khuspe, 1987; Monteuuis, Bon y Goh 1998; Monteuuis y Goh, 1999; Goh y Monteuuis, 2001; Daquinta et al., 2001), y en Costa Rica poco tiempo después mediante la clonación in vivo o en ambiente controlado (Murillo, Rojas y Badilla, 2003; Badilla, Murillo, Azofeifa, y Obando, 2003). La tecnología clonal pasó rápidamente a escala comercial (Goh y Monteuuis, 2005; Goh et al., 2007), donde la Cooperativa de
Mejoramiento Genético Forestal (GENFORES) ha continuado mejorando e impulsando desde Costa Rica en toda la región latinoamericana (Murillo et al., 2003; Murillo y Badilla, 2009; Badilla y Murillo, 2011a; 2011b; Espitia, Murillo y Castillo, 2011; Resende, Murillo y Badilla, 2018).

Los ensayos para la comprobación genética de teca requieren de varios años de observación rigurosa en campo, dado su ciclo largo de crecimiento. De manera clásica se menciona que, en una primera generación de mejoramiento genético, es importante observar durante al menos media rotación el desempeño de las accesiones, para poder establecer un ranking genético seguro (Zobel y Talbert, 1984). Numerosos trabajos científicos reportan valores de parámetros genéticos basados en ensayos de progenie en teca (Danarto y Hardiyanto, 2000; Murillo, Badilla y Araya, 2004; Callister y Collins, 2008; Murillo y Badilla, 2009; Espitia et al., 2011; Murillo et al., 2013; Resende et al., 2018). Sin embargo, pocos o casi ninguno de los estudios reporta resultados a partir de ensayos clonales y con suficiente edad en campo, que garanticen su validez (Badilla y Murillo, 2011b; Moya, Marín, Murillo y Leandro, 2013; Molina, 2017; Molina, Alfaro, Murillo, Badilla y Luján, 2019). Este estudio tiene como uno de sus propósitos determinar la mejor y más temprana edad de selección de ensayos clonales de teca.

La Cooperativa de Mejoramiento Genético Forestal (GENFORES) fue creada en el 2002 por la Escuela de Ingeniería Forestal del Instituto Tecnológico de Costa Rica, junto con un grupo de empresas forestales del país y de la región latinoamericana $(\mathrm{Mu}-$ rillo et al., 2013). En su misión se establece la conservación y el mejoramiento genético de especies forestales de interés en varios países y empresas forestales de la región. 
Por tanto, este estudio se realizó con el propósito de evaluar y seleccionar un grupo de clones de intercambio de teca, para ampliar la base genética del programa de mejoramiento para la especie en regiones secas de Costa Rica y de la región latinoamericana.

\section{Metodología}

El ensayo clonal se ubica en Pueblo Viejo, Mansión de Nicoya en la provincia de Guanacaste, donde se estableció en junio del 2006. La ubicación geográfica corresponde a $10^{\circ} 06^{\prime} 10^{\prime \prime}$ norte y $85^{\circ} 22^{\prime} 24^{\prime \prime}$ oeste; cuya Zona de Vida concuerda con bosque húmedo premontano transición a basal. El sitio se localiza a una elevación de $100 \mathrm{~m}$ sobre el nivel del mar, donde se registra una precipitación media anual de $2200 \mathrm{~mm}$ y una temperatura media de $25^{\circ} \mathrm{C}$ (Instituto Meteorológico Nacional, 2016), con un periodo seco que se prolonga desde mediados de noviembre hasta mediados de mayo. El sitio del ensayo se encuentra en un terreno levemente inclinado, con una pendiente menor a un $10 \%$, rodeado por áreas dedicadas a cultivos agrícolas, plantaciones forestales y parches de bosque secundario.

\section{Material gené- tico y diseño experimental}

Se evaluaron 31 genotipos provenientes de selecciones realizadas en la Zona Norte de Costa Rica, en los cantones de Upala (Canalete), San Carlos (Altamira y Pocosol) y
Los Chiles (San Emilio). Estos materiales fueron seleccionados por empresas miembro de la cooperativa GENFORES, en estas regiones de alta precipitación (más de 3500 $\mathrm{mm}$ al año), con predominio de suelos ácidos y con un período seco de máximo dos meses al año. Los materiales se intercambiaron con el Centro Agrícola Cantonal de Hojancha (empresa miembro y ubicada en el Pacífico seco del país), con el propósito de observar su comportamiento y poder a futuro seleccionar y ampliar su base genética. Se utilizaron dos accesiones locales como testigo: semilla comercial de rodales semilleros certificados (TB), así como semilla mejorada por polinización abierta o F2 (TS), procedente de un huerto semillero de esta misma organización en Hojancha.

Se utilizó el diseño experimental propuesto por GENFORES para evaluaciones genéticas (Murillo et al., 2004), el cual corresponde a bloques completos al azar con seis repeticiones (figura 1). En cada bloque se distribuyeron aleatoriamente 4 parejas de

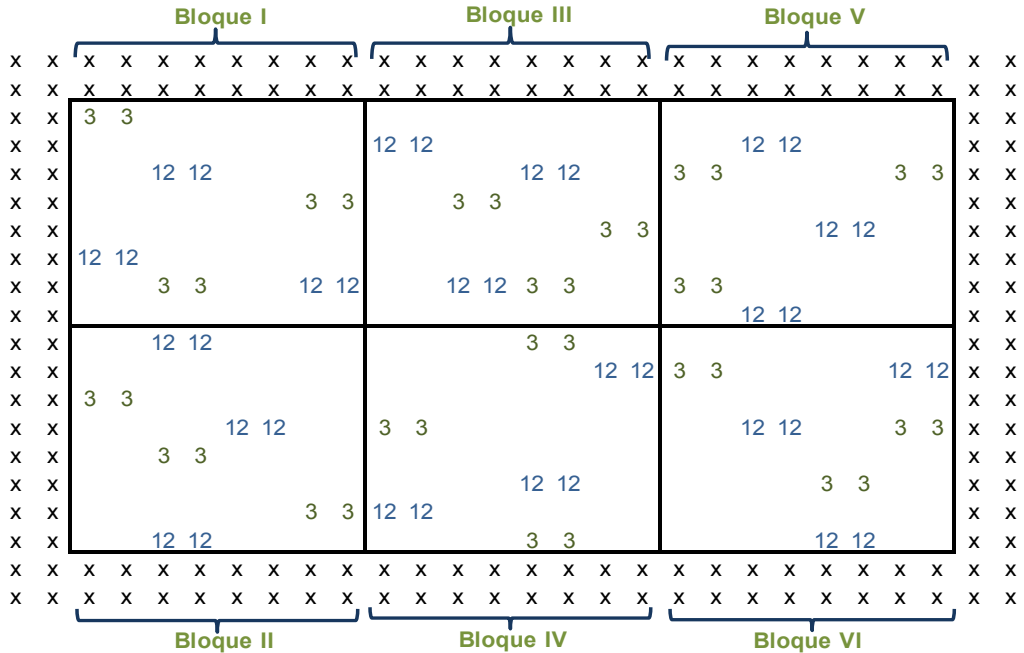

Figura 1. Diseño de GENFORES para la evaluación genética de accesiones, donde los números representan las parejas de rametos (plantas) de cada accesión, aleatoriamente distribuidas dentro de cada bloque. Extraído de Murillo et al. (2004). 
rametos (plantas) de cada clon, que conformaron una unidad experimental (parcela) de 8 plantas por clon por bloque. La densidad inicial de siembra fue de 833 árboles por hectárea a un distanciamiento de $4 \mathrm{~m}$ por $3 \mathrm{~m}$.

El sitio tuvo como uso anterior el pastoreo. No tuvo ninguna preparación inicial, más que una limpieza completa y una fertilización inicial de $50 \mathrm{~g}$ al hoyo del fertilizante 10-30-10. Durante toda la vida del ensayo se tuvo un excelente control de malezas.

El ensayo recibió un raleo del $50 \%$ a los cinco años de edad, en el cual se eliminó el árbol con peor desempeño dentro de cada pareja de rametos. De modo que la estructura genética de la población evaluada se mantuvo intacta. Es decir, todos los clones continuaron en pie y mantuvieron entre $3 \mathrm{y}$ 4 plantas por bloque. Posteriormente, a los 8 años, se realizó un segundo raleo en donde se eliminó un $25 \%$ de los árboles de menor desempeño de cada uno de los clones en cada bloque. Con esto se logró nuevamente que la estructura genética del ensayo se mantuviera completa, brindando datos por más años y resolviendo el desafío de cómo manejar la densidad y competencia dentro del ensayo.

\section{Organización y análisis de los datos de campo}

El ensayo fue evaluado en siete ocasiones $(3,25 ; 4,4 ; 5,9 ; 8,9 ; 9,7 ; 11,8$ y 12,9 años de edad), donde se midió en cada árbol el DAP, se estimó visualmente el número de trozas comerciales por árbol $(2,5 \mathrm{~m}$ de longitud) y se calificó la calidad de cada troza comercial en una escala de 1 a 4, de acuerdo con la metodología propuesta por Murillo y Badilla (2004). Donde el valor de "1" corresponde a la troza sin defectos y de la más alta calidad y potencial para aserrío, mientras que un valor de " 4 " se le asigna a la troza sin valor comercial para aserrío o exclusiva para leña.

Cada una de las siete mediciones en el tiempo fue debidamente codificada y organizada en hojas de Excel. A partir de los diámetros recolectados y el número de trozas comerciales por árbol, se estimó el volumen comercial hasta un diámetro mínimo de $5 \mathrm{~cm}$, calculado con el programa Avalúos Forestales (Murillo y Badilla 2011, versión 2019), que utiliza un algoritmo de conicidad específico para teca.

Con la información de la calidad de las trozas de cada árbol, se obtuvo una variable general denominada 'calidad del árbol', tomando en cuenta los valores de calidad de cada una de sus trozas, como se observa en la ecuación 1 :

Calidad general del árbol $=T 1 * 0,40+$
\[ T 2 * 0,30+T 3 * 0,20+T 4 * 0,10 \]

$T 2 * 0,30+T 3 * 0,20+T 4 * 0,10$

Donde: T1 corresponde al valor de calidad asignado a la troza 1, T2 es la calidad de la troza 2, sucesivamente hasta la T4 para la calidad de la troza 4. Los coeficientes que multiplican cada troza corresponden al peso económico de cada una de ellas, según su posición dentro del árbol. A menor altura en el fuste, mayor importancia económica y mayor participación en el volumen comercial de todo el árbol (Murillo y Badilla, 2004).

En vista de que el valor de calidad general del árbol es un número fraccionado que oscila entre 1 y 4 , se procedió a transformarlo a una escala de 1 a 100, para mayor facilidad de interpretación y análisis:

Calidad $(\%)=100 *\left[1-\left(\frac{\text { calidad general }-1}{3}\right)\right]$

Una vez organizadas las bases de datos con todas las variables, se procedió con su análisis estadístico. Para esto se utilizó el software Selegen versión 2012, 
desarrollado para análisis de datos genéticos por Resende (2016), el cual se basa en el método de Máxima Verosimilitud Lineal Restringida (REML, por sus siglas en inglés) y en el de Mejor Predicción Linear No Sesgada (BLUP, siglas en inglés). Este programa permite un mejor análisis de datos no balanceados, producto de la mortalidad de árboles en los ensayos, que permite la obtención de los componentes de varianza y parámetros genéticos de la población con un menor sesgo.

El análisis de los datos de los clones se realizó con base en el modelo 2 de Selegen, específico para ensayos clonales no emparentados, en una sola localidad, varias plantas por parcela:

$$
y=X r+Z g+W p+e
$$

Donde " $y$ " es el vector de datos, "r" es el vector del efecto de repetición (asumido como fijo) y sumado a la media general, "g" es el vector del efecto genético total (asumido como aleatorio), "p" es el vector de los efectos de parcela y "e" es el vector del término del error o residuo (aleatorio). Las letras mayúsculas representan las matrices de incidencia de estos efectos (Resende, 2016). Con base en este modelo se analizó cada una de las 6 edades, para cada uno de los tres caracteres (DAP, calidad del árbol y volumen comercial).

Con los datos se realizó adicionalmente, un análisis de correlación genética (r) (ligamiento genético entre caracteres) entre los caracteres evaluados a los 12.9 años de edad, que fue la edad de selección utilizada. Para su obtención, Selegen realiza previamente un procedimiento de estimación y eliminación de los efectos ambientales en los datos, para utilizar únicamente los valores genéticos de cada uno de los individuos y proceder a estimar su correlación.
La selección de las mejores accesiones se realizó con base en la variable volumen comercial, por resultar ser la de mayor heredabilidad, estabilidad en el tiempo e importancia económica. Con el valor genético promedio de los mejores 10 clones del ensayo, se estimó el diferencial de selección (S) y la ganancia genética a obtener, con relación al testigo semilla comercial sin mejorar (rodal semillero). Para este cálculo se utilizó el valor de la heredabilidad media clonal y la ganancia genética se estimó mediante la ecuación: $\mathrm{GG}=\mathrm{S} * \mathrm{~h}^{2}$ media clonal (Resende, et al. 2018).

Finalmente, se realizó un análisis de desvianza con ayuda del software Selegen (análogo al análisis de varianza), con el que se logra determinar la existencia de diferencias significativas entre clones, así como la significancia de la interacción bloque x clon (parcela). Este análisis se realizó para la edad de 12,9 años (donde se alcanzó la mayor heredabilidad) y para la variable volumen comercial, por ser la de mayor impacto económico.

\section{Resultados}

Parte importante en el análisis de datos es la veracidad o certeza de la información obtenida. Selegen genera un parámetro que denomina 'exactitud o precisión de los datos' (escala de 0,0 a 1,0), como se muestra en la tabla 1, importante para decidir sobre la confiabilidad de los resultados. Pueden observarse valores muy altos de exactitud en general ( $r a ̂ a>0,7$ Resende, 2016) para todas las siete edades y los tres caracteres evaluados, que se estabilizan a partir de los 4,4 años de edad, con cambios leves de los parámetros a través del tiempo. La mayor precisión para las tres variables simultáneamente se alcanza y estabiliza a partir de 8,9 años. 
Tabla 1

Valores de exactitud (râa) en la estimación de parámetros genéticos, para tres caracteres de importancia económica, evaluados a siete edades, en un ensayo clonal de Tectona grandis en Pueblo Viejo de Nicoya, Pacifico seco de Costa Rica

\begin{tabular}{cccc}
\hline $\begin{array}{c}\text { Edad } \\
\text { (años) }\end{array}$ & Diámetro & Calidad & $\begin{array}{c}\text { Volumen } \\
\text { comercial }\end{array}$ \\
\hline $\mathbf{3 , 2}$ & 0,65 & 0,33 & 0,63 \\
$\mathbf{4 , 4}$ & 0,89 & 0,88 & 0,75 \\
$\mathbf{5 , 9}$ & 0,93 & 0,68 & 0,87 \\
$\mathbf{8 , 9}$ & 0,89 & 0,82 & 0,86 \\
$\mathbf{9 , 7}$ & 0,86 & 0,77 & 0,58 \\
$\mathbf{1 1 , 7}$ & 0,89 & 0,79 & 0,82 \\
$\mathbf{1 2 , 9}$ & 0,90 & 0,79 & 0,85 \\
\hline
\end{tabular}

Nota: Fuente propia de la investigación.

En la tabla 2, se muestran en detalle los valores de los parámetros genéticos más importantes para las variables DAP y volumen comercial. Puede observarse que, para el DAP y el volumen comercial a los 8,9 años de edad, se alcanzan los valores más altos de ambas heredabilidades, así como de su coeficiente de variación genética. Pero sufren una ligera caída a los 5,9 años y a los 9,7 años de edad (tabla 2). Se observa también que, en ambos casos, todos los parámetros vuelven a recuperarse dos años después de esa caída, como se observa en las mediciones de 8,9 y 11,8 años de edad.

La calidad del árbol registró los valores más altos en todos sus parámetros genéticos a la temprana edad de los 4,4 años. Luego sufre una baja en todos los valores en la medición de los 5,9 años. A partir de esta edad empieza a recuperarse lentamente con el tiempo. La calidad en general registró valores inferiores de heredabilidad individual y media clonal con respecto al DAP y al volumen comercial en todos los años de medición (tabla 3).

Tabla 2

Parámetros genéticos para el DAP (izquierda) y para el volumen comercial (derecha), en 31 clones de Tectona grandis, evaluados en siete edades en Pueblo Viejo de Nicoya, Pacifico seco de Costa Rica

\begin{tabular}{ccccccc}
\hline \multirow{2}{*}{ Edad (años) } & \multicolumn{3}{c}{ Diámetro $(\mathbf{c m})$} & \multicolumn{3}{c}{ Volumen comercial $\left(\mathbf{m}^{\mathbf{3}}\right)$} \\
\cline { 2 - 7 } & $\mathbf{\mathbf { h } ^ { 2 } \mathbf { g }}$ & $\mathbf{h}^{\mathbf{2}} \mathbf{m c}$ & $\mathbf{C V g i} \mathbf{0}$ & $\mathbf{h}^{2} \mathbf{g}$ & $\mathbf{h}^{2} \mathbf{m c}$ & $\mathbf{C V g i} \mathbf{0}$ \\
\hline $\mathbf{3 , 2}$ & 0,022 & 0,43 & 2,85 & 0,024 & 0,51 & 8,69 \\
$\mathbf{4 , 4}$ & 0,120 & 0,80 & 3,51 & 0,043 & 0,56 & 5,30 \\
$\mathbf{5 , 9}$ & 0,063 & 0,73 & 2,40 & 0,028 & 0,54 & 3,90 \\
$\mathbf{8 , 9}$ & 0,120 & 0,82 & 3,03 & 0,075 & 0,74 & 5,62 \\
$\mathbf{9 , 7}$ & 0,092 & 0,73 & 2,67 & 0,020 & 0.34 & 2,84 \\
$\mathbf{1 1 , 8}$ & 0,110 & 0,79 & 3,06 & 0,065 & 0,68 & 5,20 \\
$\mathbf{1 2 , 9}$ & 0,110 & 0,80 & 3,31 & 0,076 & 0,73 & 6,00 \\
\hline
\end{tabular}

Nota: Fuente propia de la investigación.

Tabla 3

Parámetros genéticos para la variable calidad del fuste comercial, en un ensayo clonal de 31 genotipos de Tectona grandis, en siete mediciones en Mansión, Nicoya, Costa Rica

\begin{tabular}{cccc}
\hline Edad (meses) & $\mathbf{h}^{\mathbf{2}} \mathbf{g}$ & $\mathbf{h}^{\mathbf{2}} \mathbf{m c}$ & $\mathbf{C V g i} \%$ \\
\hline 3,2 & 0,003 & 0,11 & 0,81 \\
4,4 & 0,112 & 0,77 & 5,81 \\
5,9 & 0,004 & 0,12 & 0,30 \\
$\mathbf{8 , 9}$ & 0,056 & 0,67 & 2,90 \\
$\mathbf{9 , 7}$ & 0,054 & 0,59 & 2,88 \\
$\mathbf{1 1 , 8}$ & 0,062 & 0,62 & 3,10 \\
$\mathbf{1 2 , 9}$ & 0,063 & 0,63 & 3,11 \\
\hline
\end{tabular}

Nota: Fuente propia de la investigación. 
En la tabla 4 se muestran las correlaciones genéticas entre las principales variables del ensayo clonal, a la edad más temprana posible y donde se alcanza la máxima precisión de estimación. Con esto se logra reducir el tiempo de evaluación y selección de los mejores materiales genéticos de 12,9 a 8,9 años. Puede observarse que hubo una correlación débil o no significativa entre la variable calidad y las dos variables de producción (DAP y volumen comercial).

Tabla 4

Correlaciones genéticas entre las tres principales variables, en un ensayo clonal de 31 genotipos de Tectona grandis, a los

8,9 años de edad en Mansión, Nicoya, Costa Rica.

\begin{tabular}{lcc}
\hline & Calidad & $\begin{array}{c}\text { Volumen } \\
\text { comercial }\end{array}$ \\
\hline DAP & 0,370 & 0,723 \\
Calidad & 1 & 0,339 \\
Volumen Comercial & & 1 \\
\hline
\end{tabular}

Nota: Fuente propia de la investigación.
En la figura 2 se muestra el ranking genético para el volumen comercial a los 12,9 años de edad, que mostró una alta consistencia con el ranking genético de las mediciones anteriores. Puede observarse que los testigos utilizados que provienen de huerto semillero (TS) y de rodal semillero (TB) se posicionaron por debajo del promedio de los clones. La semilla mejorada genéticamente (F2) de huerto semillero se ubicó en la posición 20 del ranking genético de 33 posiciones. Mientras que la del rodal semillero se ubicó en la última posición. Se puede observar que el límite de confianza inferior de los primeros 8 clones no se traslapa con el límite superior de confianza de la semilla comercial testigo de rodal semillero, que se localizan al final del ranking, lo cual evidencia diferencias significativas entre ambos grupos.

El promedio del valor genético del volumen comercial de los clones fue de $0,864 \mathrm{~m}^{3}$ por individuo a los 12,9 años de

Figura 2. Ranking del valor genético del volumen comercial $\left(\mathrm{m}^{3}\right)$ estimado a los 12,9 años para 31 genotipos y 2 testigos comerciales de Tectona grandis en Mansión, Nicoya, Costa Rica

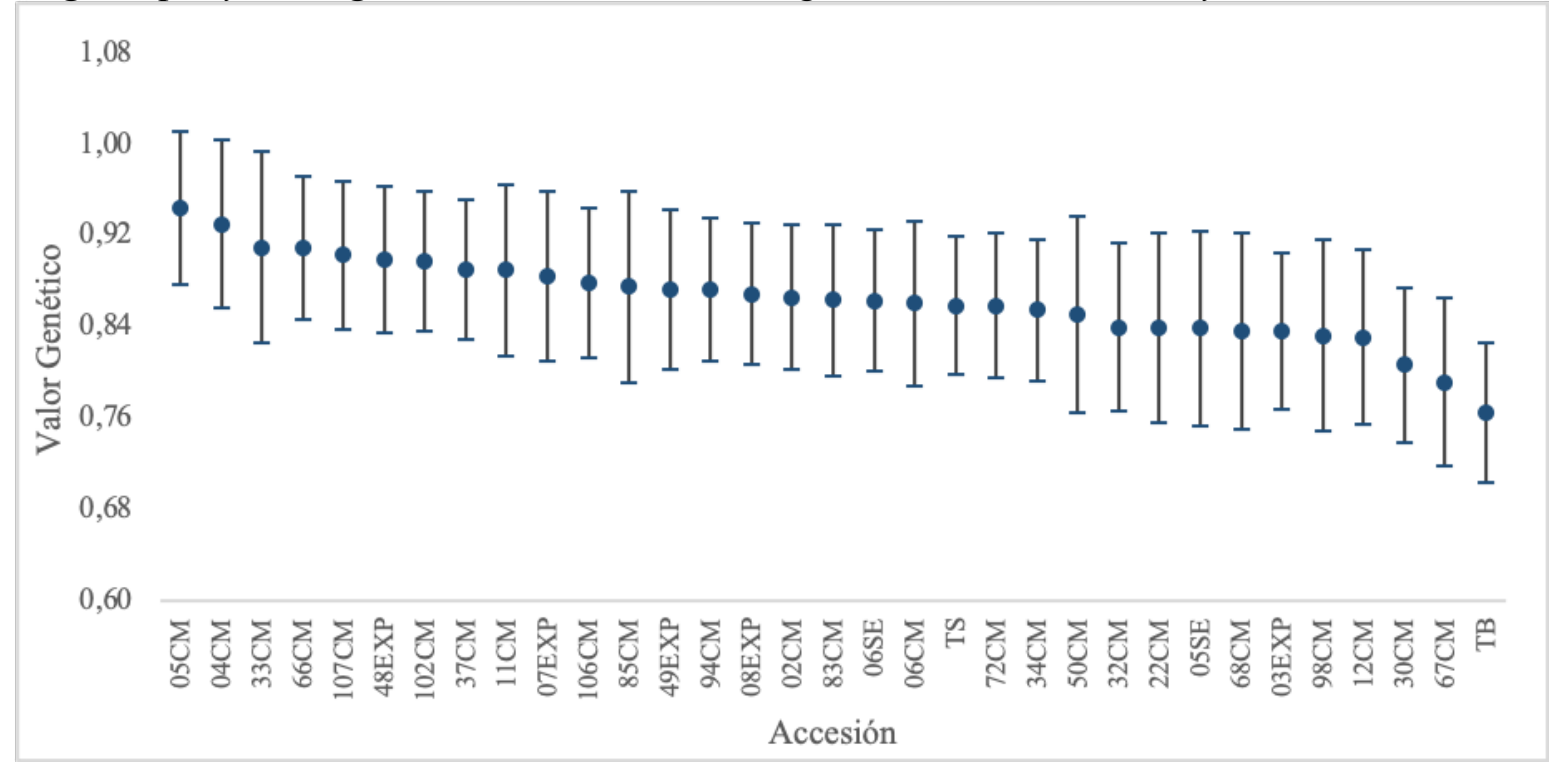

Nota: Fuente propia de la investigación. 
edad. Mientras que los dos testigos del ensayo obtuvieron $0,765 \mathrm{~m}^{3}$ para la semilla de rodal semillero (sin mejoramiento genético) y de $0,858 \mathrm{~m}^{3}$ para la semilla mejorada o F2 de huerto semillero. Si se relaciona el valor promedio de los mejores 10 clones $(0,884$ $\mathrm{m}^{3}$ ) contra el testigo semilla comercial sin mejorar $\left(0,765 \mathrm{~m}^{3}\right)$, el diferencial de selección (S) es ahora de $15,5 \%$. Al estimarse la ganancia genética esperada, se convierte en un aumento de un $11,3 \%$ en volumen comercial a los 12,9 años. Si se calcula la ganancia genética con respecto al testigo de semilla mejorada (F2), el diferencial de selección es ahora de un 3\% y la ganancia genética de $2,2 \%$ en volumen comercial a esa edad de referencia. Con respecto al crecimiento del DAP, el ensayo registró $28 \mathrm{~cm}$ en promedio a los 12,9 años, que implica un IMA de $2,21 \mathrm{~cm} /$ año.

El análisis de desvianza corrobora la existencia de diferencias significativas al 0,999\% entre clones, pero no así en la interacción BloquexClon, tal y como se muestra en la tabla 5. El componente de varianza genético y la heredabilidad también resultaron significativamente diferentes de cero.

\section{Discusión}

La exactitud (râa), tal y como se muestra en la tabla 1, es un estadístico que estima la correlación entre los valores genéticos estimados y los valores genéticos verdaderos de los individuos. A mayor exactitud en la evaluación de un individuo, mayor será la confianza en su evaluación y en su valor genético estimado (Resende $e t$ al., 2018). También puede emplearse como una medida asociada a la precisión en la selección, que depende de la heredabilidad del carácter, de la calidad y cantidad de información utilizada en la predicción de los valores genéticos. Por tanto, cuanto mayor sea su valor, mejor predicción del valor genético verdadero. Valores superiores a $r a \hat{a}=$ 0,7 pueden considerarse como de muy alta confiabilidad (Resende, 2016).

En la tabla 1 se observa que en los tres caracteres y en casi todas las edades, los valores de exactitud que sustentan esta investigación resultaron ser muy altos, estables y confiables a partir de los 4,4 años de edad. Dichos resultados sugieren que la especie alcanza una importante expresión genética a partir de los 4 años de edad en estos caracteres, y son similares a los reportados en otros trabajos con ensayos clonales de teca en el país (Badilla y Murillo, 2011b; Guzmán, Moya y Murillo, 2017; Molina, 2017).

El efecto de la edad en la expresión genética es claramente observable en el registro de la tabla 2. Queda establecido que los valores de heredabilidad y el coeficiente

Tabla 5

Análisis de desvianza para el volumen comercial en un ensayo clonal a los 12,9 años de edad, con 31 genotipos y 2 testigos de Tectona grandis en Mansión, Nicoya, Costa Rica

\begin{tabular}{|c|c|c|c|c|c|}
\hline Efecto & Desvianza & $\begin{array}{c}\text { Chi cuadrado } \\
\text { (LRT) }\end{array}$ & Significancia & Comp. varianza & $\begin{array}{l}\text { Coeficiente de } \\
\text { determinación }\end{array}$ \\
\hline Clon & $-1209,70$ & 10,91 & $* * *$ & $\mathrm{Vg}=0,00268 * * *$ & $\mathrm{~h}^{2} \mathrm{~g}=0,0763 * * *$ \\
\hline BloquexClon & $-1220,34$ & 0,27 & ns & $\begin{array}{l}\text { VBloquexClon }= \\
0,00074 \mathrm{~ns}\end{array}$ & $\begin{array}{l}\mathrm{c}^{2} \text { BloquexClon }= \\
0,0209 \mathrm{~ns}\end{array}$ \\
\hline Modelo Completo & $-1220,61$ & & & $\mathrm{Ve}=0,0317$ & \\
\hline
\end{tabular}


de variación genética tienden a mejorar con la edad (este patrón es más evidente en el carácter volumen comercial que en el DAP). Sin embargo, se observa un patrón de altibajos que se registra en la medición de los 5,9 años y los 9,7 años de edad. En el año 5 el ensayo fue raleado por lo bajo en aproximadamente un $50 \%$, que claramente afectó negativamente los parámetros genéticos. Al eliminarse una cantidad importante de individuos de la población de mejoramiento, es de esperar una disminución en la variabilidad general del ensayo, tal y como se registra en el coeficiente de variación genética $(\mathrm{CVg} \%)$. El impacto en la disminución de los árboles se refleja con mayor evidencia en la variable calidad (tabla 3), que alcanza su valor más alto en heredabilidad a los 4,4 años, debido a que las características cualitativas se expresan a más temprana edad que las variables de crecimiento como el volumen comercial (Murillo y Badilla, 2004). El raleo se enfoca precisamente en eliminar los individuos de las categorías inferiores de calidad (categorías 3 y 4), lo cual explica claramente su impacto en un parámetro como el coeficiente de variación genética (CVgi\%, tabla 3). Mientras que un segundo raleo de aproximadamente un $33 \%$ de los árboles fue ejecutado después del año 8 , que podría explicar la reducción de los parámetros genéticos en las variables cuantitativas (DAP y volumen comercial), detectada en la medición de los 9,7 años. A partir de esta edad, se puede observar en las mediciones de los 11,8 años y 12,9 años una recuperación en todos los parámetros genéticos, como es de esperar. Los árboles remanentes continúan creciendo y expresando su potencial genético en un espacio mayor que les liberó el raleo.

Este efecto del raleo en ensayos genéticos se ha reportado en otros trabajos
(Pavlotzky y Murillo, 2012; Pavlotzky y Murillo, 2013; Molina, 2017), donde la disminución de árboles de menor diámetro y calidad en la población reduce considerablemente el coeficiente de variación genética de las variables como el DAP. Sin embargo, es necesario señalar que, a pesar de eliminarse el $50 \%$ de los individuos con peor desempeño o adaptabilidad en el ensayo genético, cada clon mantiene en pie el otro $50 \%$ con sus mejores representantes. Lo cual genera un análisis más robusto desde el punto de vista de comparación y de estimación de parámetros genéticos.

\section{Edad de selección y ganancia genética}

Se eligió como edad de selección los 8,9 años, debido a que fue la edad más temprana donde se alcanzaron valores altos de heredabilidad, así como el mayor coeficiente de variación genética en las tres variables de producción investigadas (tablas 2 y 3 ). Esto es particularmente importante en variables como el volumen comercial, donde participa gran parte del genoma y requiere, por tanto, mayor tiempo para observar su expresión. En la literatura clásica sobre mejoramiento genético forestal (Zobel y Talbert, 1984), se menciona que en programas jóvenes de mejoramiento es relevante esperar al menos hasta la mitad de la rotación para poder realizar una selección segura de genotipos. La rotación en teca ocurre entre los 18 y 20 años, por lo que una edad segura sería entre los 9 y 10 años. Sin embargo, la edad de selección podría ocurrir mucho antes, dependiendo de qué información se desee obtener. En este trabajo se analizó la posición de los mejores 10 clones en el ranking genético del volumen comercial a distintas edades, donde se observó que las variaciones fueron menores a partir de los 8,9 años de edad, con 
excepción del testigo semilla mejorada, que fue cayendo desde la posición 13 hasta la 20 conforme aumentó la edad de las mediciones. Sin embargo, la estimación de la ganancia genética con la selección de los 10 mejores clones sí fue aumentando progresivamente con la edad, debido a que el diferencial de selección (S) aumentó con respecto a los dos testigos. El segundo testigo (semilla comercial de rodal semillero) fue perdiendo posiciones en el ranking genético hasta ubicarse en la última posición a partir de la medición a los 11 años de edad. La mayor estabilidad de los clones en el tiempo puede explicarse por su composición genética, constituida por un solo individuo o genotipo. Por su parte, la constitución genética de los dos testigos corresponde a una mezcla de semillas de rodal y de huerto semillero, con una variación mucho mayor que tiende a comportarse muy diferente a los clones con el paso del tiempo; un comportamiento de promedio de sus genotipos. Estos resultados ponen en evidencia la importancia de la edad en la seguridad de la selección de genotipos en teca.

El análisis de correlaciones genéticas busca identificar un posible efecto o impacto indirecto de la selección por una variable de interés, como el volumen comercial, en alguna otra variable relevante, como alguna característica cualitativa del fuste o de alguna propiedad de la madera. La correlación genética puede sugerir una relación compleja entre variables a nivel genómico, una cercanía espacial a nivel cromosómico, que puede traer dificultades en un programa de mejoramiento genético (Resende et al., 2018). Por lo que es importante explorar este tipo de asociaciones entre caracteres de interés. La correlación genética entre las variables (tabla 4) muestra que fue débil entre la calidad y las dos variables cuantitativas (DAP y volumen comercial). Resultados diferentes han sido reportados en otros trabajos con teca (Kjaer, Kaosa-ard y Suangtho, 2000; Callister y Collins, 2008), donde se determinó una correlación positiva entre el volumen del árbol y la rectitud del tallo. Sin embargo, la variable calidad del árbol de este estudio está compuesta por la expresión de varios caracteres cualitativos del fuste y ramas, que se resumen en una calificación visual con valores de 1 a 4 (Murillo y Badilla, 2004). Por tanto, es una mezcla de varios caracteres, bajo una influencia ambiental variable, cuyo control genético colectivo (heredabilidad) resultó ser baja, tal y como se registra en la tabla 4. Una mejor estimación podría obtenerse en próximos trabajos si se correlacionaran los caracteres cuantitativos con caracteres específicos cualitativos, como rectitud de fuste, ausencia de gambas o aletones, dominancia apical, ausencia de bifurcaciones, ausencia de rama gruesa, entre otros. Estos resultados corroboran una asociación débil entre las variables cuantitativas y la variable calidad utilizada en esta investigación.

Los resultados del ranking genético del volumen comercial a los 12,9 años muestran el mejor desempeño de un grupo de 19 clones con respecto a los dos testigos. Estos resultados reafirman un cumplimiento de los objetivos del estudio, donde se logra identificar un buen comportamiento y adaptación de un grupo de clones, introducidos desde las zonas húmedas del norte a la región del Pacífico seco del país. Debe recordarse que los clones introducidos son árboles plus de primera generación, es decir, sin mejoramiento genético (Vallejos, Badilla, Picado y Murillo, 2010). En contraste, la semilla de huerto semillero es producida por polinización abierta y aleatoria entre un grupo selecto de árboles plus; por tanto, semilla mejorada genéticamente o F2. Es 
decir, que 19 clones introducidos desde otra región del país hayan superado a la semilla mejorada local testigo debe considerarse un resultado muy positivo, desde el punto de vista de poder ampliar la base genética del programa de mejora de la teca en regiones secas del país. Una comparación más justa, al mismo nivel de mejoramiento genético, podría hacerse con respecto al segundo testigo, que corresponde a semilla comercial de rodal semillero. En este caso todos los clones superaron ampliamente el desempeño de la semilla, que se ubicó en la última posición del ranking. De esta comparación se obtuvo una ganancia genética de un $11 \%$ en volumen comercial, que es significativa y de alto impacto. Con un aumento en crecimiento de esta magnitud, una plantación clonal de teca lograría reducir en 1 a 2 años el turno de rotación en el Pacífico seco del país (Murillo et al., 2019).

\section{Conclusiones}

Se logró identificar un grupo de 19 clones de teca de alto rendimiento y buena adaptación a la región del Pacífico seco del país. La selección de los mejores 10 clones introducidos desde la Zona Norte podría generar una ganancia genética de un $11 \%$ en volumen comercial a los 12,9 años de edad.

Con un aumento en crecimiento de esta magnitud, una plantación clonal de teca lograría reducir en cerca de 2 años el turno de rotación en el Pacífico seco del país.

Una plantación de teca en la península de Nicoya, con los mejores 10 clones determinados en esta investigación, podría alcanzar un volumen comercial de $0,88 \mathrm{~m}^{3} /$ árbol y un DAP de $28 \mathrm{~cm}$ a los 12,9 años de edad.

A los 8,9 años se registró la mayor heredabilidad individual y la heredabilidad media clonal para el DAP $\left(\mathrm{h}^{2} \mathrm{~g}=0,12\right.$ $\left.\mathrm{y} \mathrm{h}^{2} \mathrm{mc}=0,82\right)$ y para el volumen comercial $\left(h^{2} \mathrm{~g}=0,075 \mathrm{y} \mathrm{h}^{2} \mathrm{mc}=0,74\right)$.

\section{Agradecimientos}

A la Cooperativa Internacional de Mejoramiento Genético Forestal (GENFORES) por su apoyo en la realización de este trabajo. Al Centro Agrícola Cantonal de Hojancha y a los hermanos Rodríguez por todo el apoyo en el establecimiento, manejo e investigaciones desarrolladas en el ensayo clonal de teca.

\section{Funding}

FundaTEC GENFORES 03-401.

\section{Conflicto de intereses}

Los autores declaran no tener algún conflicto de interés.

\section{Declaración de la contribución de los autores}

Todos los autores afirmamos que se leyó y aprobó la versión final de este artículo.

El porcentaje total de contribución para la conceptualización, preparación y corrección de este artículo fue el siguiente: Y.B.V. $60 \%$ y O.M.G. $40 \%$.

\section{Declaración de disponibilidad de los datos}

Los datos que respaldan los resultados de este estudio serán puestos a disposición por el autor correspondiente Y.B.V., previa solicitud razonable. 


\section{Referencias}

Badilla, Y. \& Murillo, O. (2011a). Evaluación del comportamiento de clones de teca (Tectona grandis) en Costa Rica. En $V$ Congreso Forestal Latinoamericano. Universidad de $\mathrm{La}$ Molina, Lima, Perú.

Badilla, Y. \& Murillo, O. (2011b). Avances en el mejoramiento genético de la teca en GENFORES, Costa Rica. En Conferencia Forestal Internacional: Bosques plantados de teca Teaknet, 31 oct - 3 nov. San José, Costa Rica.

Badilla, Y., Murillo, O., Azofeifa, M. \& Obando, G. (2003). Avances en reforestación clonal en Costa Rica. En V Congreso Forestal Nacional. San José, Costa Rica.

Béhaghel, I. (1999). Etat des plantations de teck (Tectona grandis L.f.) dans le monde. The state of teak (Tectona grandis) plantations worldwide. Bois et Forêts des Tropiques, (262), 6-18.

Callister, A. N. \& Collins, S. L. (2008). Genetic parameter estimates in a clonally replicated progeny test of teak (Tectona grandis Linn. f.). Tree Genetics \& Genomes, 4(2), 237.

Cameron, A. L. (1966). Genetic improvement of teak in New Guinea. Australian Forestry, 30(1), 76-87.

Danarto, S. \& Hardiyanto, E. B. (2000). Results of the progeny test of teak at 12 years of age at Jember, East Java. Potential and opportunities in marketing and trade of plantation teak: challenge for the new millennium. Proceeding of the Third Regional Seminar on Teak, 249-253. Faculty of Forestry Gadjah Mada University, Yogyakarta, Indonesia.

Daquinta, M., Ramos, L., Capote, I., Lezcano, Y., Rodríguez, R., Trina, D. \& Escalona, M. (2001). Micropropagación de la teca (Tectona grandis L.f.). Revista Forestal Centroamericana, 35, 25-28.

Dupuy, B. \& Verhaegen, D. (1993). Le teck de plantation Tectona grandis en Côte d'Ivoire. Bois \& Forets des Tropiques, 235, 9-24.

Espitia, M., Murillo, O. \& Castillo, C. (2011). Ganancia genética esperada en teca (Tectona grandis L.f.) en Córdoba. Colombia forestal, 14(1), 81-93.

Fonseca González, W. (2004). Manual para productores de teca (Tectona grandis L.f.) en Costa Rica. Heredia, Costa Rica: FONAFIFO.
Goh, D. K. S. \& Monteuuis, O. (2005). Rationale for developing intensive teak clonal plantations, with special reference to Sabah. Bois et Forêts des Tropiques, 285, 5-15.

Goh, D. K. S., Chaix, G., Bailleres, H. \& Monteuuis, O. (2007). Mass production and quality control of teak clones for tropical plantations: The Yayasan Sabah Group and Forestry Department of Cirad Joint Project as a case study. Bois et Forêts des Tropiques, 293, 65-77.

Goh, D. K. \& Monteuuis, O. (2001). Production of tissue cultured teak: the plant biotechnology laboratory experience. Potential and opportunities in marketing and trade of plantation teak: challenge for the new millennium. Proceeding of the Third Regional Seminar on Teak. Faculty of Forestry Gadjah Mada University, Yogyakarta, Indonesia.

Gram, K. \& Larsen, C. S. (1958). The flowering of teak (Tectona grandis) in aspects of tree breeding, based on observations in Thailand. Natural History Bulletin of the Siam Society NHBSS. The Siam Society (FAO/TSC-60/3.2).

Guzmán, N., Moya, R. \& Murillo, O. (2017). Evaluation of bent trees in juvenile teak (Tectona grandis L.f.) plantations in Costa Rica: Effects on tree morphology and wood properties. Forests, 8(3), 79. https://doi:10.3390/f8030079

Instituto Meteorológico Nacional (2016). Datos climáticos, geoportal. https://www.imn.ac.cr/ web/imn/inicio

Keiding, H., Wellendorf, H. \& Lauridsen, E. B. (1986). Evaluation of an international series of teak provenance trials. Horsholm, Dinamarca: Forskningscentret for Skov \& Landskab

Keiding, H. (1960). Budding and grafting of teak. Documento, Subcomisión de la Teca, tercera sesión, Nueva Delhi, India, 1960. FAO/TSC 60/3.3.

Keogh, R. (1979). Does teak have a future in tropical America? Unasylva, 31, 13-19.

Keogh, R. (1980). Teak (Tectona grandis) provenances of the Caribbean, Central America, Venezuela and Colombia. En: IUFRO Meeting, Working Group S1.07.09. Río Piedras, Puerto Rico: IUFRO.

Kjaer, E.D., Kaosa-ard, A. \& Suangtho, V. (2000). Domestication of teak through tree improvement. Options, possible gains and critical factors. En: T. Enters \& C.T.S. Nair (Eds.). Site, technology and productivity of teak plantations, FORSPA N²4/2000, Teaknet Publication $N^{\circ} 3$, 161-189. Bangkok: Teaknet. 
Mascarenhas, A.F., Kendurkar, S.V., Gupta, P.K., Khuspe, S.S. \& Agrawal, D.C. (1987). Teak. En Bonga J.M. \& Durzan, D.J. (Eds.). Cell and Tissue Culture in Forestry, Vol. 3. Dordrecht, Países Bajos: Martinus Nijhoff Publishers.

Molina-Quesada, S., Alfaro, C., Murillo, O., Badilla, Y. \& Luján, R. (2019). Evaluación del comportamiento de clones de Tectona grandis L.f. en suelos vertisoles de la península de Nicoya, Costa Rica. Revista Forestal Mesoamericana Kurú, 16(38), 24-34. https://doi. org/10.18845/rfmk.v16i38.3993

Molina-Segura, F. (2017). Comportamiento de clones de teca (Tectona grandis Linn.) a los 4, 5 años en Upala, Zona Norte de Costa Rica. Cartago: Instituto Tecnológico de Costa Rica.

Monteuuis O. \& Goh D. (1999). About the use of clones in teak. Bois et Forêts des Tropiques, 261, 28-38.

Monteuuis, O., Bon, M. C. \& Goh, D. K. (1998). Teak propagation by in vitro culture. Bois et Forets des Tropiques. 256, 1-11.

Moya, R., Marín, J. D., Murillo, O. \& Leandro, L. (2013). Wood physical properties, color, decay resistance and stiffness in Tectona grandis clones with evidence of genetic control. Silvae Genetica, 62(1-6), 142-152. https:// doi.org/10.1515/sg-2013-0019

Murillo, O., \& Badilla, Y. (2011). Programa para determinar la calidad y el valor de la plantación forestal. Cartago, Costa Rica: Instituto Tecnológico de Costa Rica.

Murillo, O. \& Badilla, Y. (2004). Evaluación de la calidad y estimación del valor en pie de la plantación forestal. Cartago, Costa Rica: Instituto Tecnológico de Costa Rica.

Murillo, O. \& Badilla, Y. (2009). Mejora genética de la teca: avances y tendencias en los últimos 10 años. En I Congreso Internacional del Cultivo de Teca (pp. 16-17). Universidad de Quevedo, Ecuador.

Murillo, O., Badilla, Y. \& Araya, E. (2004). Breeding teak in Costa Rica. En IUFRO Meeting. Forest Genetics and Genomics. Charleston, Carolina del Norte, USA.

Murillo, O., de Resende, M. D. V., Badilla, Y. y Gamboa, J. P. (2019). Genotype by environment interaction and teak (Tectona grandis L.f.) selection in Costa Rica. Silvae Genetica, 68(1), 116-121. https://doi.org/10.2478/ sg-2019-0020
Murillo, O., Rojas, J. L. \& Badilla, Y. (2003). Reforestación clonal. Cartago: Instituto Tecnológico de Costa Rica.

Murillo, O., Wright, J., Monteuuis, O. \& Montenegro, F. (2013). Capítulo 6: Mejoramiento genético de la teca en América Latina. En: De Camino, R. y Morales, J.P. (Eds.). Las plantaciones de teca en América Latina: Mitos $y$ realidades (pp. 86-111). Turrialba, Costa Rica: CATIE.

Pavlotzky, B. \& Murillo, O. (2012). Ganancia genética esperada en Acacia mangium en Los Chiles, Costa Rica. Agronomía Mesoamericana, 23(1), 1-13.

Pavlotzky, B. \& Murillo, O. (2013). Ganancia genética esperada en Acacia mangium en San Carlos, Costa Rica. Forestal Mesoamericana, 10(24), 14-25. https://doi.org/10.18845/rfmk. v10i24.1320

Resende, M., Murillo, O. \& Badilla, Y. (2018). Genética cuantitativa y selección en el mejoramiento forestal. Cartago: Editorial Tecnológica de Costa Rica.

Resende, M. (2016). Software Selegen-REML/BLUP: a useful tool for plant breeding. Crop Breeding and Applied Biotechnology, 16, 330-339. https://doi. org/10.1590/1984-70332016v16n4a49

Richens, R. H. (1945). Forest tree breeding and genetics. Cambridge: Imperial Bureau of Plant Breeding and Genetics.

Schnell, G. S. \& Paludzyszyn, E. (2010). Cenário da silvicultura de teca e perspectivas para o melhoramento genético. Pesquisa Florestal Brasileira, 30(63), 217-230. https:// doi:10.4336/2010.pfb.30.63.217

Vallejos, J., Badilla, Y., Picado, F. \& Murillo, O. (2010). Metodología para la selección e incorporación de árboles plus en programas de mejoramiento genético forestal. Agronomía Costarricense, 34(1), 105-119.

Zobel, B. \& Talbert, J. (1984). Applied Forest Tree Improvement. Nueva York: John Wiley and Sons. 


\section{(c) (i) $(9) \Theta$}

Selección clonal de Tectona grandis L. f. para el Pacífico seco de Costa Rica (Yorleny Badilla-Valverde - Olman Murillo-Gamboa) Uniciencia is protected by Attribution-NonCommercial-NoDerivs 3.0 Unported (CC BY-NC-ND 3.0) 